\title{
Tests based on sample partial autocorrelations
}

\author{
SIMON F. KU
}

The theme of this thesis is a study of limit theory involving sample partial autocorrelations in general random processes with a view to statistical inference. The results clarify the well-known theory ("Quenouille's Tests") in the setting of classical autoregressive (AR) stationary processes, and extend to non-stationary processes of asymptotically stationary kind, such as simple branching (Bienaymé-Galton-Watson) processes with immigration (BPI). The limit theory of sample partial autocorrelations is then sketched for general stochastic processes, not restricted even to autoregressive moving-average (ARMA) type processes. This is demonstrated through a detailed study of ARMA(1,1)type processes, in which a joint limit distribution of normed sample partial autocorrelations is explicit in terms of the joint limit distribution of normed sample autocorrelations, assuming this last obtains. This explicit result is then applied to some bilinear models $(B L)$, in particular $\mathrm{BL}(1,0,1,1)$, in the setting of asymptotic stationarity.

The sequence of above topics leads to a (largely empirical) study of related aspects of $\operatorname{ARMA}(2,1)$-type processes.

The concept of partial autocorrelation is also exploited in the so-called peaks test, under the assumption that readings actually come from a stationary Gaussian ARMA process, as alternative hypothesis to the null hypothesis of randomness.

In Chapter 1, we start with the classical stationary $\operatorname{AR}(p)$ processes, summarise the well-known results relating to the sample partial autocorrelations, and motivate the generalisation to the $\operatorname{AR}(p)$-type random processes which have the same autocorrelation structure as that of stationary $\operatorname{AR}(p)$ processes. We then show that the existence of a vector limit distribution for normed sample autocorrelations implies a vector limit distribution for normed sample partial autocorrelations and we display the relationship between the limit distributions. The motivation of the paper Mills and Seneta [4] is crucial to Chapter 1. The main theorem obtained, when considered for the classical $\operatorname{AR}(p)$ case, clarifies Quenouille's celebrated results $[6,7]$. Analogy and distinction are also made between the stationary and non-stationary situations. This chapter forms the content of the paper $\mathrm{Ku}$ and Seneta [3].

\section{Received 19th January, 1998}

Thesis submitted to The University of Sydney, February 1997. Degree approved, October 1997. Supervisor: Professor Eugene Seneta.

Copyright Clearance Centre, Inc. Serial-fee code: 0004-9729/98 \$A2.00+0.00. 
In Chapter 2, we look at limit theory of sample partial autocorrelations in a random process. The methodology developed in this chapter is general and does not require the process to be second-order stationary, nor does it require normality of the limit distribution of sample autocorrelations.

In Chapter 3, the principle of Chapter 2 is applied to a process of ARMA-type, for which the moving-average (MA) component, in addition to the AR component, is taken into account. Thus this chapter continues, but in a different sense, the study initiated in Chapter 1. The MA part causes considerable complexity even in the simplest case of this kind, namely, ARMA(1,1)-type processes. We are able to obtain an explicit form of a limit distribution based on the sample partial autocorrelations of such processes. For the ARMA(1,1)-type case, the approach is motivated by that of Mills and Seneta [4]. An application of limit results for $\operatorname{ARMA}(1,1)$-type processes to certain bilinear models is also discussed. Chapters 2 and 3 form the paper of $\mathrm{Ku}$ [1].

In Chapter 4, motivated by the theory developed in Chapter 1, which is applicable to a simple BPI, we investigate a sum of two independent simple subcritical BPI's. This results in an $\operatorname{ARMA}(2,1)$-type process. Investigation of processes of this kind is a natural consequence of the focus on ARMA(1,1)-type processes of Chapter 3 . The research is motivated by issues raised (Suresh, Chandra and Koteeswaran) in [9], underpinning whose work is the fundamental paper of Venkataraman [10]. Much of this investigation is empirical, and a number of tables produced by simulations are used to illustrate the conclusions.

In Chapter 5, we use the idea of partial correlation to demonstrate the computability of the autocovariance function of peaks occurring in a random process. The calculation is related to evaluation of orthant probabilities for a zero mean quadrivariate normal distribution with correlation structure new to the literature. These autocovariances are needed for the evaluation of the variance of the total number of peaks in a sample. The reduction formula of Plackett [5] plays a key role. Once the actual variance is obtained, the statistical analysis can be carried out using an appropriate Central Limit Theorem, which is derived. One of the motivating papers for the work of this chapter is Stigler [8], whose approach the author of this thesis has extended to more general stationary ARMA processes as alternatives to the hypothesis of random sequence. This chapter forms a major part of the paper published as Ku and Seneta [2].

The chapters have been written so that it is possible to read each of: Chapter 1, Chapters 2 and 3, Chapter 4, Chapter 5 independently, despite the connecting theme and sequential development.

\section{REFERENCES}

[1] S. $\mathrm{Ku}$, 'Limited distribution of sample partial autocorrelations: a matrix approach', Stochastic Process. Appl. 72 (1997), 121-143. 
[2] S. Ku and E. Seneta, 'The number of peaks in a stationary sample and orthant probabilities', J. Time Ser. Anal. 15 (1994), 385-403.

[3] S. Ku and E. Seneta, 'Quenouille-type theorem on autocorrelations', Ann. Inst. Statist. Math. 48 (1996), 621-630.

[4] T.M. Mills and E. Seneta, 'Goodness-of-fit for a branching process with immigration using sample partial autocorrelations', Stochastic Process. Appl. 33 (1989), 151-161.

[5] R.L. Plackett, 'A reduction formula for normal multivariate integrals', Biometrika 41 (1954), 351-360.

[6] M.H. Quenouille, 'A large-sample test for the goodness of fit of autoregressive schemes', J. Roy. Statist. Soc. Ser. A 110 (1947), 123-129.

[7] M.H. Quenouille, 'Approximate tests of correlation in time-series', J. Roy. Statist. Soc. Ser. $B 11$ (1949), 68-84.

[8] S.M. Stigler, 'Estimating serial correlation by visual inspection of diagnostic plots', Amer. Statist. 40 (1986), 111-116.

[9] K. Suresh Chandra and P. Koteeswaran, 'Inference on superimposed subcritical Galton-Watson processes with immigration', Ann. Inst. Statist. Math. 38 (1986), 311-318.

[10] K.N. Venkataraman, 'A time series approach to the study of the simple subcritical Galton-Watson process with immigration', Adv. Appl. Probab. 14 (1982), 1-20.

School of Mathematics and Statistics

The University of Sydney

NSW 2006 Australia

e-mail: simonk@maths.usyd.edu.au 